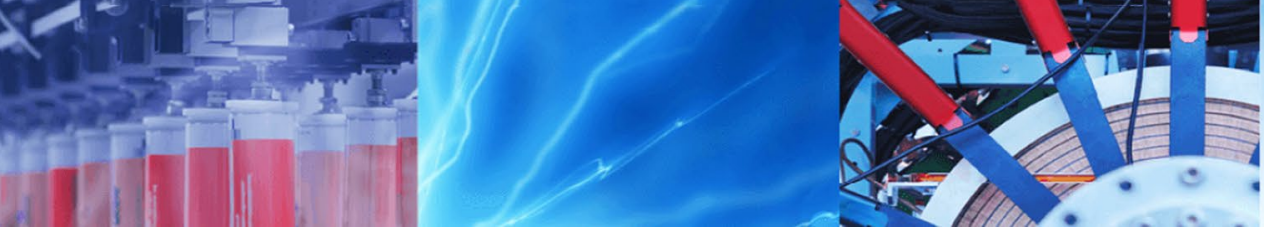

Research Article

\title{
Incorporation of lipid nanosystems containing omega-3 fatty acids and resveratrol in textile substrates for wound healing and anti-inflammatory applications
}

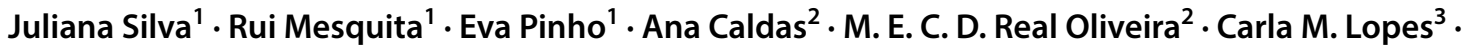 \\ Marlene Lúcio ${ }^{2,4} \cdot$ Graça Soares $^{1}$ (D)
}

๑) Springer Nature Switzerland AG 2019

\begin{abstract}
In the present work, lipid nanosystems containing omega-3 fatty acid (nanostructured lipid carriers, NLCs) or omega-3 fatty acid and resveratrol (liposomes) were developed to improve cotton textile substrates as dressings with anti-inflammatory properties for wound healing applications. Lipid nanosystems were incorporated into woven, non-woven and knitted cotton substrates by exhaustion and impregnation. Based on physical-chemical characterization of the textile substrates, the textile structure and type of lipid nanosystems dictated the adsorption efficiency. In the case of NLCs, the woven substrate functionalized by exhaustion had a higher omega-3 release being the most promising for wound dressing application. Whereas for liposomes, the most adequate textile was the cationized knitted fabric functionalized by impregnation, that showed a more prolonged release profile of resveratrol.
\end{abstract}

Keywords Textiles lipid nanosystems · Liposomes · Nanostructured lipid carriers (NLCs) · Wound healing · Antiinflammatory effects

\section{Introduction}

Wound healing is a complex physiological process involving several phases, including inflammation, proliferation and regeneration. Textiles are still the most used material to assist this complex process. Nowadays, there is a demand for smart wound dressings that, besides basic mechanical protection, must improve the healing process by interacting with the wound through the release of bioactive molecules and/or by maintaining the favorable conditions for the re-establishment of skin integrity and homeostasis [1]. Omega-3 fatty acids are natural bioactive compounds with proven anti-inflammatory activity [2].
Despite the literature reported benefits of omega- 3 fatty acids, they still have some issues regarding the hydrophobicity, high propensity for oxidation, unpleasant smell and taste and low bioavailability. Lipid nanosystems have been pointed out as a successful strategy to overcome some of those limitations $[3,4]$.

The use of bioactive compounds with anti-inflammatory action such as resveratrol (RSV) have also reported benefic effects in wound healing processes $[5,6]$. One of RSV's limitations is its rapid metabolization by the human body [7] and also its propensity to suffer oxidation and produce the inactive cis-isomer. To overcome this drawback, the encapsulation of RSV in liposomes, that might

Electronic supplementary material The online version of this article (https://doi.org/10.1007/s42452-019-1049-4) contains supplementary material, which is available to authorized users.

$\triangle$ Graça Soares, gmbs@det.uminho.pt | ${ }^{1}$ Centre for Textile Science and Technology (2C2T), University of Minho, Guimarães, Portugal. ${ }^{2}$ Centre of Physics of University of Minho and Porto (CF-UM-UP), University of Minho, Braga, Portugal. ${ }^{3}$ Fernando Pessoa Energy, Environment, and Health Research Unit/Biomedical Research Center (FP-ENAS/CEBIMED), Faculty of Health Sciences, Fernando Pessoa University, Porto, Portugal. ${ }^{4}$ Centre of Molecular and Environmental Biology (CBMA), University of Minho, Braga, Portugal. 
provide protection against early degradation and metabolism, is a strategy that has been investigated $[8,9]$.

Based on the galenic (i.e. the smell of fish oil) and stability (i.e. oxidation of both omega-3 and RSV) issues, the main goal of this study was to functionalize textile substrates with lipid nanosystems containing omega-3 (NLCs) or omega- 3 and RSV (liposomes) which are intended to be used in wound healing.

Liposomes and nanostructured lipid carriers (NLCs) were produced and used to functionalize cotton textile substrates, by exhaustion and impregnation processes. The materials were characterized based on their Fouriertransform infrared spectroscopy with attenuated total reflectance (FTIR-ATR) and differential scanning calorimeter (DSC) profiles, as well as air and water vapor permeability properties. The bioactive release profiles from lipidbased nanosystems were also studied.

To our knowledge, this is the first study regarding textile substrates functionalized with lipid nanosystems containing RSV and omega-3.

\section{Materials and methods}

\subsection{Materials}

Precirol ${ }^{\circledR}$ ATO 5 (glyceryl palmitostearate), was donated by Gattefossé (France). Omega-3, composed by $70 \%$ of eicosapentaenoic acid (EPA), $18 \%$ of a- linolenic acid (ALA) and $12 \%$ of docosahexaenoic acid (DHA), was acquired from MyProtein (Portugal). Tween ${ }^{\circledR} 80$ was purchased from Acofarma ${ }^{\oplus}$ (Spain) and benzalkonium chloride from Merck Schudart (Germany). Hexane and resveratrol (RSV) with a purity $>99 \%$ were obtained from Sigma-Aldrich ${ }^{\oplus}$ (Portugal). 1,2-Distearoyl-sn-glycero-3-phosphocholine (DSPC) and 1,2-Dioleoyl-sn-glycero-3-phosphoethanolamine (DOPE) with a purity $>99 \%$ were acquired from NOF laboratories (Japan). Three textile substrates 100\% cotton were used: woven $(5 \times 5 \mathrm{~cm})$, knitted fabrics $(5 \times 5$ and $25 \times 40 \mathrm{~cm}$ ) and non-woven (disks with a diameter of $5.7 \mathrm{~cm}$ ).

All lipid colloidal dispersions were prepared using ultrapure water, obtained from a Milli-Q purification system (Millipore Corporation).

\subsection{Production of lipid nanosystems}

NLC dispersions were prepared by ultrasound technique, as reported by Silva et al. [10]. The lipid phase was composed by Precirol ${ }^{\circledR}$ ATO 5 (7 wt\%) and omega-3 (3wt\%) as solid and liquid lipids, respectively. The aqueous phase contained Tween ${ }^{\circledast} 80$ (2.5 wt\%) and benzalkonium chloride $(0.5 \mathrm{wt} \%)$, that act as surfactants, and ultrapure water (87 wt\%).

Liposomes containing DSPC (0.4 wt\%), DOPE (0.2 wt\%) and omega-3 $(0.1 \mathrm{wt} \%)$ were prepared by the lipid film hydration method using ultrapure water $(99.3 \mathrm{wt} \%)$ followed by extrusion $[8,9]$. RSV $(200 \mu \mathrm{M}$ final concentration, regarded as suitable for obtaining anti-inflammatory effect $[10,11])$ was incorporated in the preformed liposomes by an incubation method as previously reported $[8,9]$. For sake of clarity the lipid nanosystems will be simply referred as NLCs (designating NLCs containing omega-3 fatty acid) and Liposomes (designating liposomes containing omega-3 fatty acid and RSV).

\subsection{Characterization of the lipid nanosystems}

\subsubsection{Physical-chemical characterization and stability in storage conditions}

To avoid the multi-scattering effect of light, lipid nanosystems were diluted with purified water (1:10) and where characterized regarding their size and distribution (measured by the polydispersity index, PDI) by dynamic light scattering in a Zetasizer Nano ZS laser scattering device (Malvern Panalytical, Paralab, Portugal) at a backscattering angle of $173^{\circ}$. Zeta-potential (ZP) of the lipid nanosystems was evaluated by electrophoretic light scattering (ELS) in a Zetasizer Nano ZS (Malvern Panalytical, Paralab, Portugal).

To evaluate physical stability of the lipid nanosystems under storage conditions (at $4{ }^{\circ} \mathrm{C}$ ) the abovementioned physical-chemical characterization was made during a time span of two months.

\subsubsection{In vitro cellular evaluation of anti-inflammatory effect}

The excess production of nitric oxide (NO) has been associated with acute and chronic inflammation and consequent tissue damage $[12,13]$. Therefore, the anti-inflammatory properties of the lipid nanosystems was evaluated in vitro using the murine macrophage (RAW 264.7) cell line, according to the method previously described by Sobral et al. [14]. The results were expressed in percentages of inhibition of NO production in comparison with the negative control (100\%).

\subsection{Functionalization of textile substrates}

Textile substrates (woven, non-woven and knitted cotton fabrics) were functionalized with NLCs and liposomes by exhaustion and/or impregnation. In the exhaustion functionalization process, textile substrates $(5 \times 5 \mathrm{~cm})$ were immersed in $5 \mathrm{~mL}$ of NLCs aqueous dispersion or 
liposomes. They were maintained for $24 \mathrm{~h}$ at room temperature (RT), under mechanical agitation (60 rpm). To dry, the textile substrates were kept at $25^{\circ} \mathrm{C}$ for $24 \mathrm{~h}$. All samples were weighted before and after functionalization.

In the impregnation functionalization process, knitted fabrics with $25 \times 40 \mathrm{~cm}$ size were immersed in $100 \mathrm{~mL}$ of each formulation and then pass through the foulard ( $1 \mathrm{~m} \mathrm{~min}^{-1}$ and 2 bar). Each impregnated fabric was wrapped in a plastic tube, covered with cling film and left at RT for $24 \mathrm{~h}$. To dry, the textile substrates were unwrapped and left at RT for $24 \mathrm{~h}$. All samples were weighted before and after functionalization.

\subsection{Characterization of the textile substrates}

\subsubsection{Chemical characterization using FTIR-ATR}

The functionalized textile substrates were characterized by FTIR-ATR, DSC and air and water vapor permeability. For the FTIR-ATR analysis, the spectra were recorded between 400 and $4000 \mathrm{~cm}^{-1}$, with increments of $4 \mathrm{~cm}^{-1}$ and 45 scans/min, in FTIR Shimadzu Iraffinity.

\subsubsection{Thermodynamic characterization using DSC}

DSC measurements were carried in liquid nitrogen atmosphere using DSC-822e instrument (Mettler Toledo). The calibration was made with indium as standard. Samples were weighed $(2.5 \pm 0.2 \mathrm{mg})$ and sealed in aluminum pans. Then, they were heated from 25 to $350{ }^{\circ} \mathrm{C}$, at a scanning rate of $10{ }^{\circ} \mathrm{C} \mathrm{min}^{-1}$. Data were treated using LAB Mettler Star SW 8.1 software (Mettler-Toledo International Inc., Swiss).

\subsubsection{Air and water vapor permeability}

The air/water vapor permeability is a basic physical property of wound dressings that may influence the wound healing process $[15,16]$, and for that reason should be investigated. Textile substrates functionalized by impregnation were used in air and water vapor permeability tests. The substrates functionalized by this technique were the only ones with the minimum dimensions required to perform these tests. Air permeability tests were carried using FX3300 instrument (TEXTEST Instruments) in accordance with BS 7209:1990, with test head $20\left(20 \mathrm{~cm}^{2}\right)$. Ten measurements were made, all in different areas of the samples, with a pressure of $200 \mathrm{~Pa}$.

Water vapor permeability tests were carried using M261 instrument (SDL) in accordance with EN ISO 9237:1995.

\subsubsection{Release of bioactive compounds from textile substrates}

To assure that the bioactive compounds encapsulated in the lipid nanocarriers have a therapeutic effect, it is necessary that they are released from the tissues'scaffolds. Therefore, it is necessary to study the release profile of such compounds. The release medium was chosen to provide the necessary chemical affinity for bioactives, so that release is simulated. RSV has moderate lipophilic character [9] and its solubilization is favored in amphiphilic media. Hence, RSV will only be released from liposomes if it has chemical affinity to the surrounding medium. As such, RSV release from liposomes was evaluated in micellar media $(\mathrm{pH}=5.5)$ simulating the hydrolipidic content and $\mathrm{pH}$ values of skin [17], mimicking the actual environment surrounding the tissue scaffold upon application on skin. Contrastingly, omega-3 is a highly lipophilic bioactive, and its release from NLC would have only been possible to lipid enriched domains of skin by fusion of the lipid nanosystems in contact with skin surface. As this situation is not easy to simulate, we have opted to use a nonpolar release medium ( $n$-hexane) which is one of the widely used lipid-like organic phase in partition coefficient studies [18]. Similarly, it has been reported the use of other nonpolar organic solvents to evaluate the in vitro release of fish oils from microcapsules [19]. The use of such solvents as release media does not mimic the in vivo release conditions but allows to provide a chemical environment that is similar to the nonpolar media encountered in high lipid content of skin where the omega-3 fatty acids will solubilize. Furthermore, the release into an organic media allows to demonstrate that the fish oils were successfully encapsulated.

The functionalized textile substrates samples $(5 \times 5 \mathrm{~cm})$ were immersed in $10 \mathrm{~mL}$ of release medium. The systems were disposed in an orbital shaker incubator at $37^{\circ} \mathrm{C}$ and $80 \mathrm{rpm}$. At predetermined timepoints, sample aliquots were withdrawn and replaced with the same volume of fresh medium to maintain the sink conditions. The amount of omega-3 or RSV released was measured by UV-Vis spectrophotometry using a UV-2501 PC spectrometer (SHIMADZU), at $\lambda_{\max }$ of $269 \mathrm{~nm}$ and $305 \mathrm{~nm}$ for assessment omega- 3 and RSV, respectively. Results were presented as cumulative percentage of released compound (omega-3 or RSV) as a function of time. 


\section{Results and discussion}

\subsection{Characterization of the lipid nanosystems}

\subsubsection{Physical-chemical characterization and stability in storage conditions}

Immediately after preparation $\left(\mathrm{t}_{\mathrm{i}}\right)$ lipid nanosystems containing RSV and omega- 3 presented adequate sizes (Table 1) for skin penetration through intercellular route of stratum corneum, since it has been established that liposomal vesicles of $50-500 \mathrm{~nm}$ are able to use this penetration route [20-22]. Polydispersity index (PDI) values provide an indication of the sizes' distribution within a nanosystem suspension. Accordingly, NLC can be considered as a fairly homogenous dispersion $(\mathrm{PDI}<0.3)$ [23-25]. Contrastingly, liposomes present higher PDI values typical of heterogenous size's distribution. Considering that it is aimed a skin topical application of the lipid nanosystems, the higher values of PDI are not so relevant and could result from the insertion of more fluid lipid components (such as omega-3 and DOPE) in a more rigid lipid matrix (composed of DSPC). This strategy originates less uniformed packed vesicles, that by being more deformable originate heterogeneous dispersions. This deformability can be however useful for cutaneous application favoring the penetration through the stratum corneum [26]. Concerning the nanosystems surface charge, evaluated in terms of zeta-potential values (ZP), the presence of the cationic surfactant (benzalkonium chloride) explained the positive charge of the developed NLC dispersions (positive ZP values in Table 1). Liposomes are negatively charged (negative ZP values in Table 1) due to the ionization of the carboxylic acid group of omega-3.

During a shelf storage period of 2 months $\left(t_{f}\right), N L C s$ remained stable in terms of mean particle size and PDI that is maintained under 0.30 . However, ZP values of NLC became less negative, which could predict loss of stability of the formulation as values of ZP lower than $-30 \mathrm{mV}$ or higher than $+30 \mathrm{mV}$ are usually required to assure colloidal stability by surface charge repulsion $[27,28]$. Despite this fact, since the charge neutralization was not followed by a great increase in the size of nanoparticles, it is expected that Tween ${ }^{\circledR} 80$ added to NLC composition might have conferred stability to the formulation being able to prevent the aggregation of nanoparticles by steric mechanisms [29]. Liposomes that were more disordered and heterogeneous when first prepared $\left(t_{i}\right)$ apparently suffered lipid packing and/or reordering during the storage. Indeed, after 2 months of storage, liposomes present lower sizes and PDI. Notwithstanding the PDI improvement, ZP values become less negative meaning that as a future prospect the formulation should be improved to include a stabilizing element which prevents liposomal aggregation (for example by coating systems with polyethylene glycol, PEG).

\subsubsection{In vitro cellular evaluation of anti-inflammatory effect}

The inflammatory response following tissue injury plays important roles both in normal and pathological healing [30]. However, recent studies also suggest that the inflammatory process should be contained as failure to do this, plays a role in the pathogenesis of non-healing wounds $[31,32]$. Accordingly, the production of excessive amounts of nitric oxide (NO) contributes to inflammation and tissue injury [33]. Regarding this rational, we hypothesized that RSV and omega-3 fatty acids can ameliorate wound healing due to an anti-inflammatory effect by inhibition of $\mathrm{NO}$ production, an effect that has been reported in previous studies $[34,35]$. NLCs presented a NO production inhibition of $64.14 \pm 2.08 \%$ demonstrating an effect of approximate magnitude of phenylethanoids (NO production inhibition of 6.3-62.3\%) that for the extent of NO production inhibition were reported to possess anti-inflammatory properties [36]. The NO production inhibition effect obtained with NLCs is also in accordance with the results obtained by Lima et al. [34]. These authors reported that high concentrations of EPA and DHA lead to NO production inhibition [34].

Liposomes presented an inferior NO production inhibition $(21.97 \pm 1.27 \%)$, which was however within the NO production inhibition effect achieved by phenylethanoids [36]. The smaller effect in this case must be also related
Table 1 Characterization of liposomes (loaded with RSV and omega-3) and NLCs (loaded with omega-3)
SN Applied Sciences

\begin{tabular}{|c|c|c|c|c|c|c|}
\hline \multirow[t]{2}{*}{ Formulation } & \multicolumn{2}{|l|}{ Size (nm) } & \multicolumn{2}{|l|}{ PDI } & \multicolumn{2}{|l|}{$\mathrm{ZP}(\mathrm{mV})$} \\
\hline & $t_{i}$ & $t_{f}$ & $t_{i}$ & $t_{f}$ & $t_{i}$ & $t_{f}$ \\
\hline Liposomes & $152 \pm 17$ & $113 \pm 11$ & $0.58 \pm 0.10$ & $0.39 \pm 0.02$ & $-26 \pm 1$ & $-19 \pm 1$ \\
\hline NLCs & $129 \pm 1$ & $135 \pm 9$ & $0.19 \pm 0.004$ & $0.24 \pm 0.007$ & $+25 \pm 1$ & $+8 \pm 1$ \\
\hline
\end{tabular}

All values represent the mean \pm standard deviation $(n=3) . t_{i}$ represents characterization immediately after preparation and $t_{f}$ represents characterization perform after storage at $4{ }^{\circ} \mathrm{C}$ for 2 months 
with the smaller concentrations of omega-3 fatty acid in this formulation.

All in all, both formulations were effective reducing NO production, which can be regarded as a promising advantage in the wound healing process.

\subsection{Functionalization of textile substrates}

Figure 1 presents the mass gain for the substrates functionalized with NLCs and lipossomes by exhaustion and impregnation techniques.

In the substrates functionalized with NLCs by exhaustion, the mass gain was significantly higher than the non-functionalized ones (control), which confirms the incorporation of NLC into the textiles tested (Fig. 1a). This incorporation is however due to the ability of the textile substrates to absorb and retain the nanosystems dispersions and it does not result from the affinity between dispersions-substrate since all textile substrates are composed exclusively of cotton fibers. Accordingly, the nonwoven fabric was the substrate with the highest mass gain, due to its higher absorption capacity (97.35\%) (Fig. 1a). No mass gain occurred in the knitted fabric functionalized with NLCs by impregnation, maybe because this method does not use a long incubation period that might be required for the retention of the nanosystems.

In all substrates functionalized with liposomes by exhaustion, there was no significant differences in the mass gain relatively to the control (Fig. 1a). Since liposomes possess a negatively charged surface (Table 1) two knitted textile substrates were tested: non-cationized and cationized (Fig. 1b), however only cationized knitted fabric impregnated with liposomes demonstrated a mass gain about $8 \%$ higher than that recorded in the control. The reasons why the cationized knitted fabric functionalized by exhaustion with liposomes did not present any mass gain are not clear at this point. One might hypothesize that the electrostatic interaction between negatively charged liposomes and the positively charged fabric tissue may induce liposomal fusion and vesicle disaggregation that motivates the loss of vesicles adsorbed to the fabric, especially during the prolonged incubation period used in exhaustion technique. Liposomal fusion onto surfaces has been described on similar incubation conditions [37, 38]. When cationized knitted fabrics are functionalized by impregnation there is not such a prolonged incubation period which might justify the successful electrostatic adsorption of liposomes to the fabric.

Figure 2 presents, as an example, the FTIR-ATR spectra obtained from knitted textile substrates functionalized by exhaustion (a) and impregnation (b). Spectra obtained by exhaustion for other substrates (woven and non-woven) are very similar and are presented as supplementary data.

All the spectra present similar vibration modes with few notorious differences, which can be justified by the common chemical moieties encountered either in the lipidic constituents of the nanosystems [39-45] or in the bioactive omega-3 [42] or even in the textile substrate [43]. The presence of the bioactive RSV, normally detected by the presence of trans olefinic bands at 986 and $964 \mathrm{~cm}^{-1}$ [44], was also not possible to assign. This is probably due to two factors: (1) the low concentration of RSV in formulation and (2) the fact of RSV being encapsulated in the liposomes thus justifying the lack of intensity of its ATRbands which strongly depend on the depth of penetration the evanescent light source. Moreover, in agreement to the current study, other reported of RSV-loaded liposomes, also did not present any differences in FTIR spectra with and without RSV [44].

Despite of the abovementioned resemblance of chemical groups, spectra of the textile substrates functionalized with NLCs (blue spectra), either by exhaustion (Fig. 2a) or impregnation (Fig. 2b), present differences in comparison with the respective controls (grey
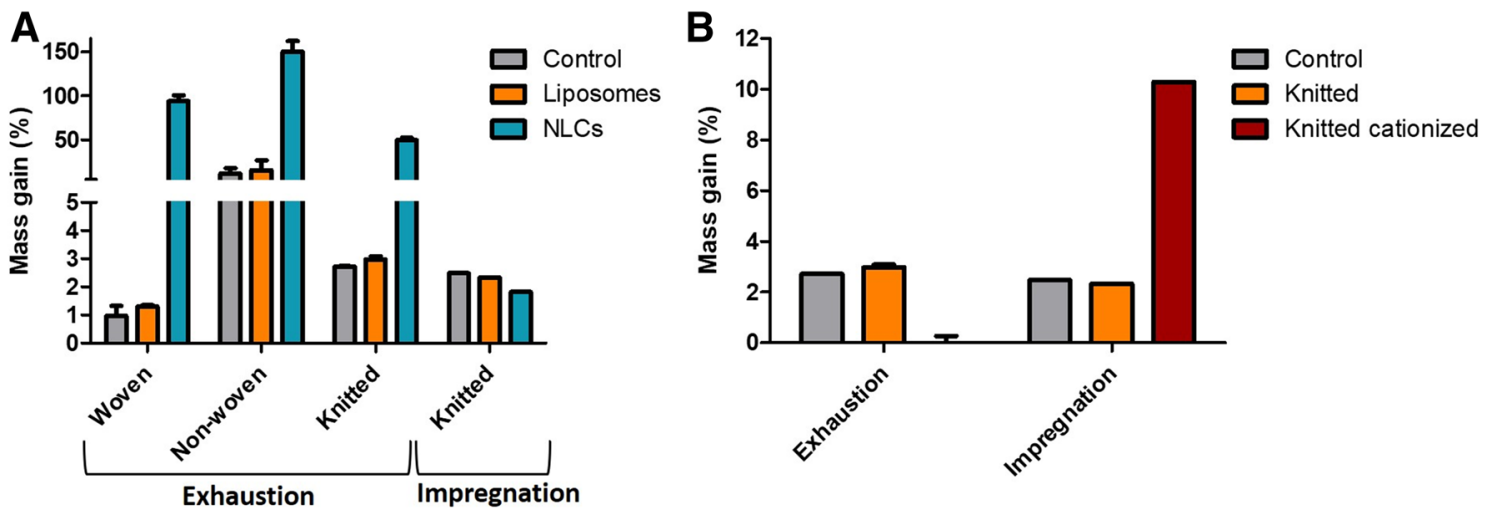

Fig. 1 a Mass gain and respective standard deviation of textile substrates functionalized with lipid nanosystems (NLCs and liposomes) using exhaustion and impregnation technique. b Mass gain of knitted substrates (cationized and non-cationized) functionalized with liposomes 


\section{A EXHAUSTION}

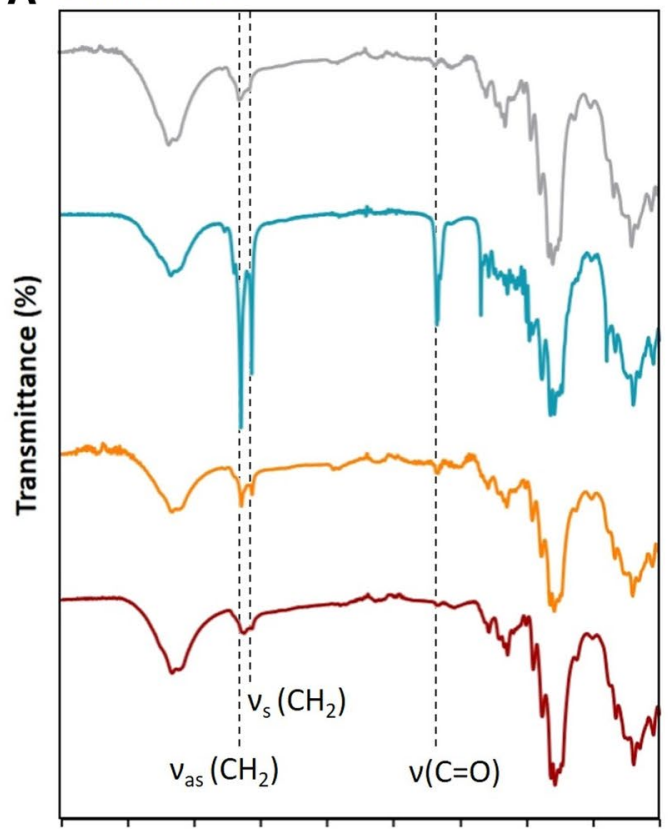

40003600320028002400200016001200800400 $\mathrm{v}\left(\mathrm{cm}^{-1}\right)$

\section{B IMPREGNATION}

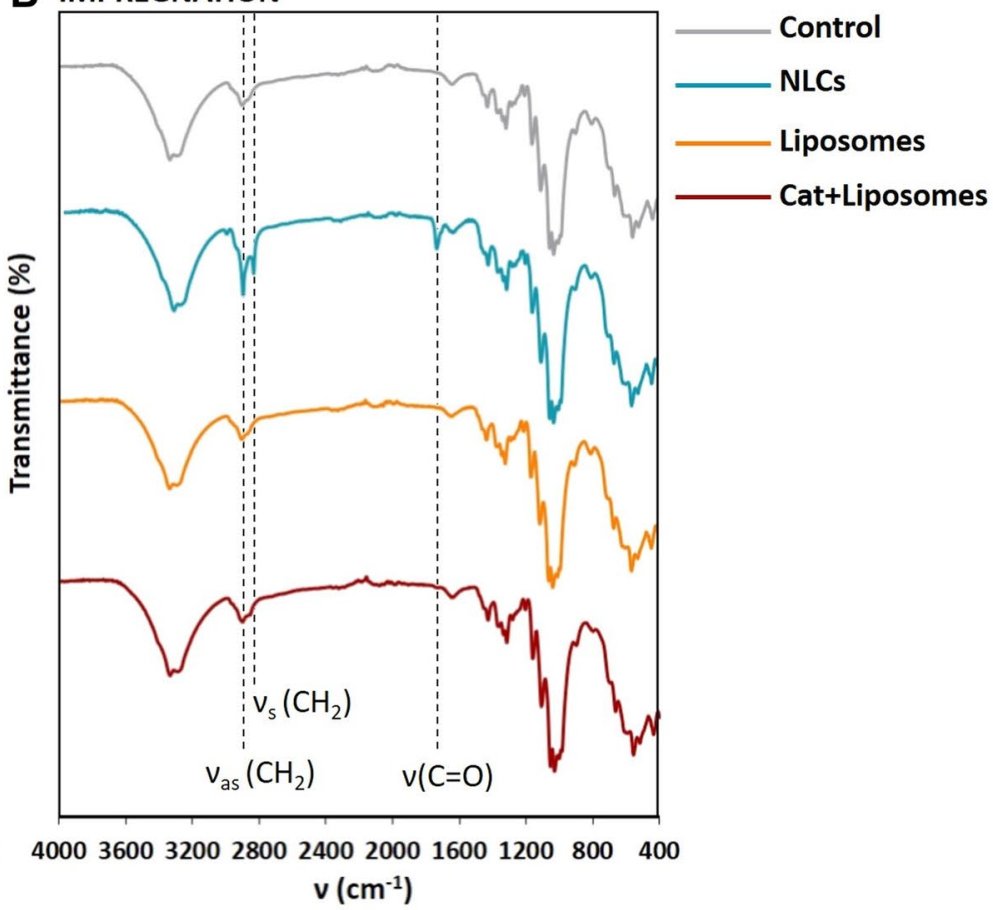

Fig. 2 FTIR-ATR transmittance spectra obtained from knitted fabrics functionalized by $\mathbf{a}$ exhaustion or $\mathbf{b}$ impregnation. Dashed lines indicate the vibration modes of $\mathrm{CH}_{2}$ and $\mathrm{C}=\mathrm{O}$. Control are the bare knitted fabric spectra (grey colour); NLCs are the spectra of knitted

spectra). The first marked difference corresponds to the appearance of more intense bands in NLCs spectra (blue spectra) at 2912 and $2850 \mathrm{~cm}^{-1}$ that can be respectively attributed to $\mathrm{CH}_{2}$ asymmetric $\left[\mathrm{v}_{\mathrm{as}}\left(\mathrm{CH}_{2}\right)\right]$ and symmetric $\left[\mathrm{v}_{s}\left(\mathrm{CH}_{2}\right)\right]$ stretching vibration modes. Although textile substrate possesses $\mathrm{CH}_{2}$ groups in its composition, these groups appear in higher number in the long aliphatic tails of fatty acids constituents of lipids (e.g. Precirol $^{\circledR}$ ATO 5) [39] or omega-3 [42], therefore originating more intense bands. The second difference noticed is a band at $1732 \mathrm{~cm}^{-1}$ characteristic of $\mathrm{C}=\mathrm{O}$ bonds that are also part of the ester region of both Precirol ${ }^{\circledR}$ ATO 5 and omega-3 $[39,42]$. The vibration modes characteristic of NLCs spectra are evident in both methods of functionalization, but are less intense in the impregnation method (Fig. 2a) which maybe related with the smaller efficiency of this method to functionalize textiles with NLCs as corroborated by the lower mass gain of the textile substrate (Fig. 1a).

As explained above, the incorporation of liposomes in the textile substrates did not shown relevant differences in FTIR-ATR spectra either due to the low concentration of liposomes or by coincidence of bands in liposomes' spectra with bands in textiles' spectra (Fig. 2a-orange spectrum). fabric functionalized with NLCs (blue colour); Liposomes are the spectra of knitted fabric functionalized with liposomes (orange colour) and Cat + liposomes are the spectra of cationized knitted fabric functionalized with liposomes (wine colour)

Figure 3 presents, as an example, the DSC thermograms obtained from knitted textile substrates functionalized by exhaustion (a) and impregnation (b). Thermograms obtained for other substrates (woven and non-woven) functionalized by exhaustion are very similar and are presented as supplementary data.

All thermograms present an endothermic transition between 53 and $60^{\circ} \mathrm{C}$. This transition is believed to be the result of water evaporation and the melting/decomposition of waxes and lipids present on the textile substrates [46]. Near the end of the thermograms, the beginning of a new endothermic transition, around $350^{\circ} \mathrm{C}$, can also be detected. This transition was also reported by Scacchetti [47] and is associated to the thermal decomposition process of cellulose.

In the thermograms of the knitted textile substrates functionalized with NLCs (blue thermogram) either by exhaustion or by impregnation, there are two endothermic transitions between 51 and $64^{\circ} \mathrm{C}$ which correspond to the endothermic transition of the cotton that occurred in the control, and to the endothermic transition of the solid lipid used (Precirol ${ }^{\circledR}$ ATO5). The endothermic transition of the solid lipid is in agreement with the obtained by Avasatthi et al. $\left(63.8^{\circ} \mathrm{C}\right)$ [48]. Wang et al. [49] demonstrated that, when Precirol ${ }^{\circledR}$ ATO5 is incorporated into NLC dispersions, 


\section{A EXHAUSTION}

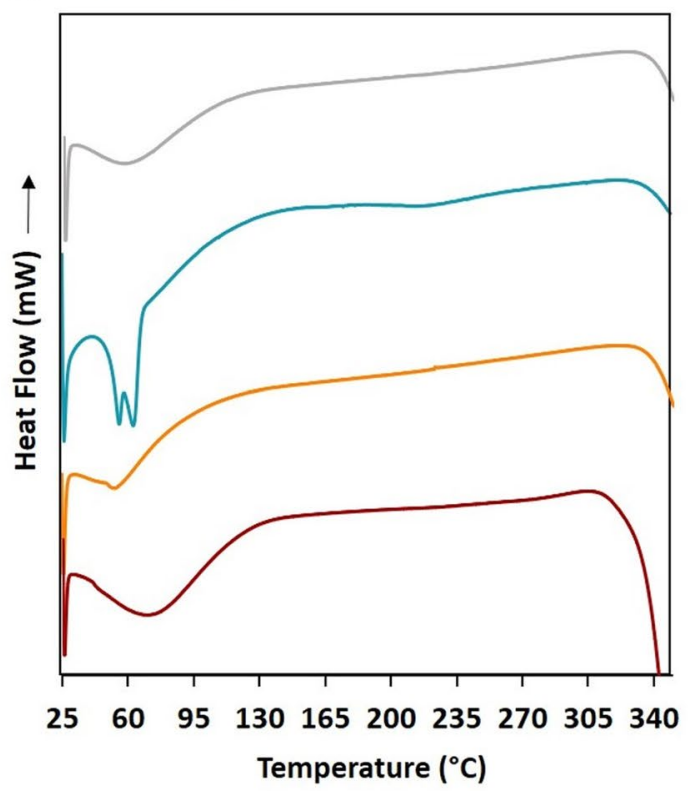

B IMPREGNATION

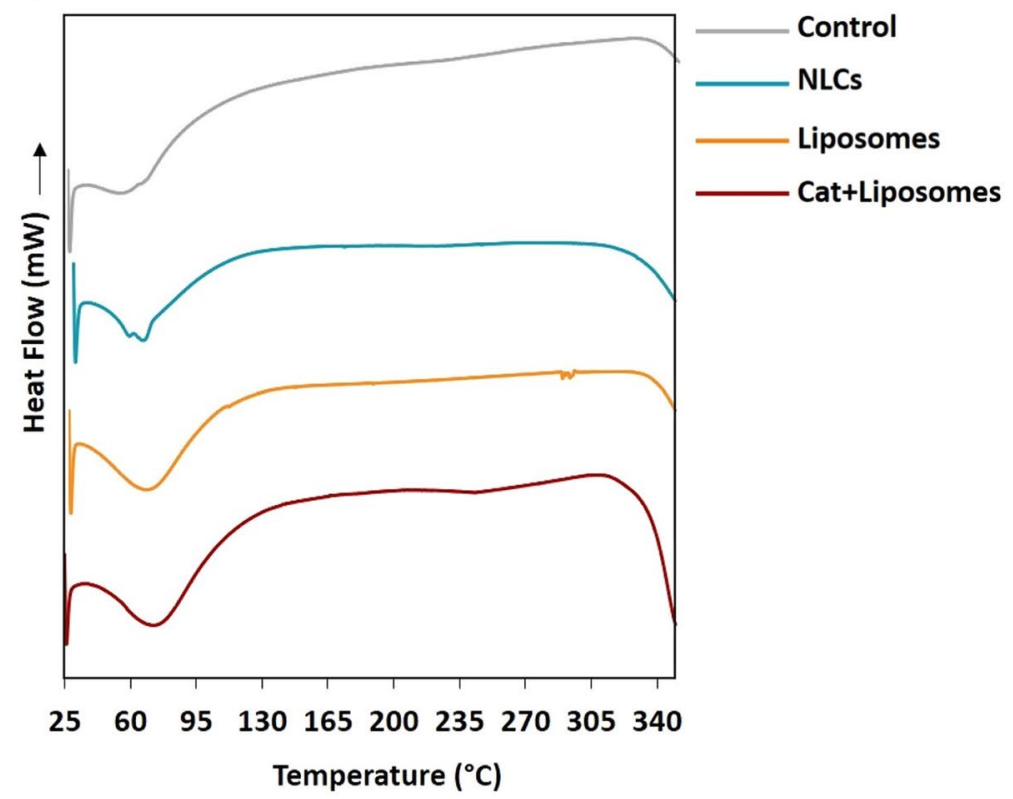

Fig. 3 DSC thermograms obtained from knitted fabrics functionalized by $\mathbf{a}$ exhaustion or $\mathbf{b}$ impregnation. Control are the bare knitted fabric spectra (grey colour); NLCs are the spectra of knitted fabric functionalized with NLCs (blue colour); Liposomes are the spectra of knitted fabric functionalized with liposomes (orange colour) and Cat + liposomes are the spectra of cationized knitted fabric functionalized with liposomes (wine colour)

bar-liposomes) did not alter the so called "breathability" of the tissue, which is important for wound healing proposes.

The impregnation of cationized knitted fabric with liposomes revealed a $13 \%$ increase in air permeability which is probably due to fiber degradation during the cationization process. The high standard-deviation associated to the air permeability measured in cationized knitted fabric $(508 \pm 49)$ confirms that the cationization process was not homogeneous and, therefore, the impregnation was not homogeneous too.

Dressings that produce a moist wound environment, thereby promoting cell migration, have long been recognized as desirable for wound healing purposes [52]. Therefore, to attain satisfactory conditions at the wound site, the functionalization of the textile substrates should not affect its water vapor permeability. In comparison with the control (Fig. $4 \mathrm{~b}$ grey bar), none of the impregnated samples have shown a significant reduction of water vapor permeability. Therefore, in wound healing, this fabric will be able to prevent dehydration of the wound, but it might not be the best choice to wounds with a high amount of exudate.

Figure 5 presents omega-3 (a) and RSV (b) release profile from textile substrates.

In the case of woven and knitted fabrics functionalized by exhaustion technique, a burst of omega- 3 release is observed in the first 4 and $2 \mathrm{~h}$, respectively (Fig. $5 \mathrm{a}$ ). In the lipid nanosystems (Fig. 4a blue bar-NLCs and orange 


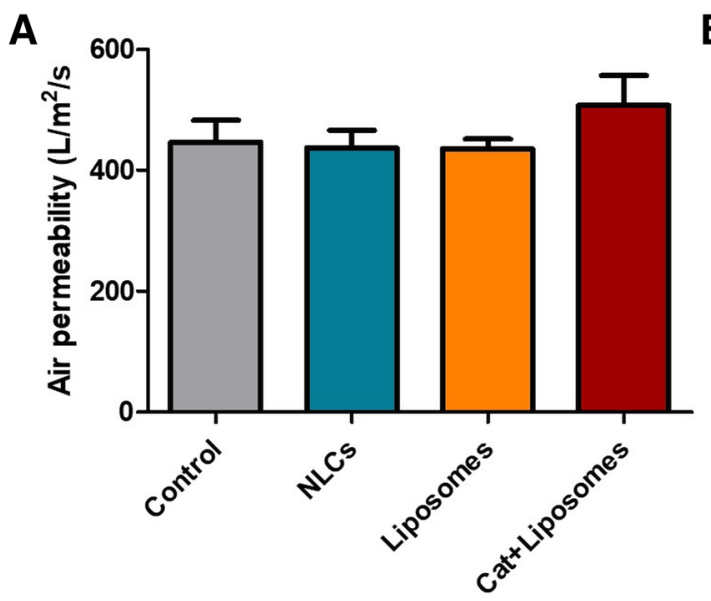

Fig. 4 Air permeability (a) and water vapor permeability (b) of knitted fabric functionalized by impregnation. Control are the bare knitted fabric spectra (grey colour); NLCs are the spectra of knitted fabric functionalized with NLCs (blue colour); Liposomes are the

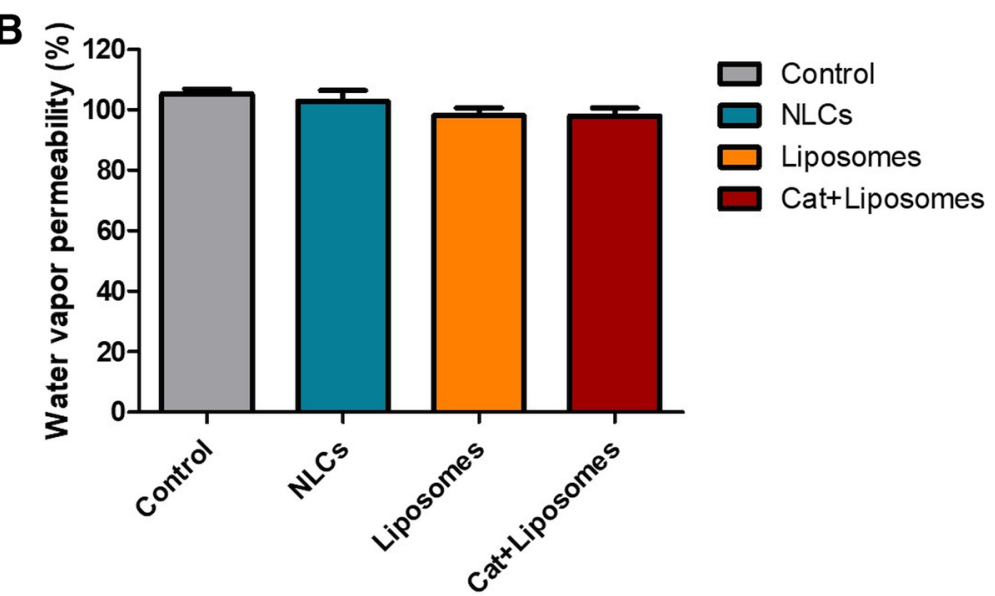

spectra of knitted fabric functionalized with liposomes (orange colour) and Cat + liposomes are the spectra of cationized knitted fabric functionalized with liposomes (wine colour)
Fig. 5 Omega-3 (a) and RSV (b) cumulative release during a $24 \mathrm{~h}$ period

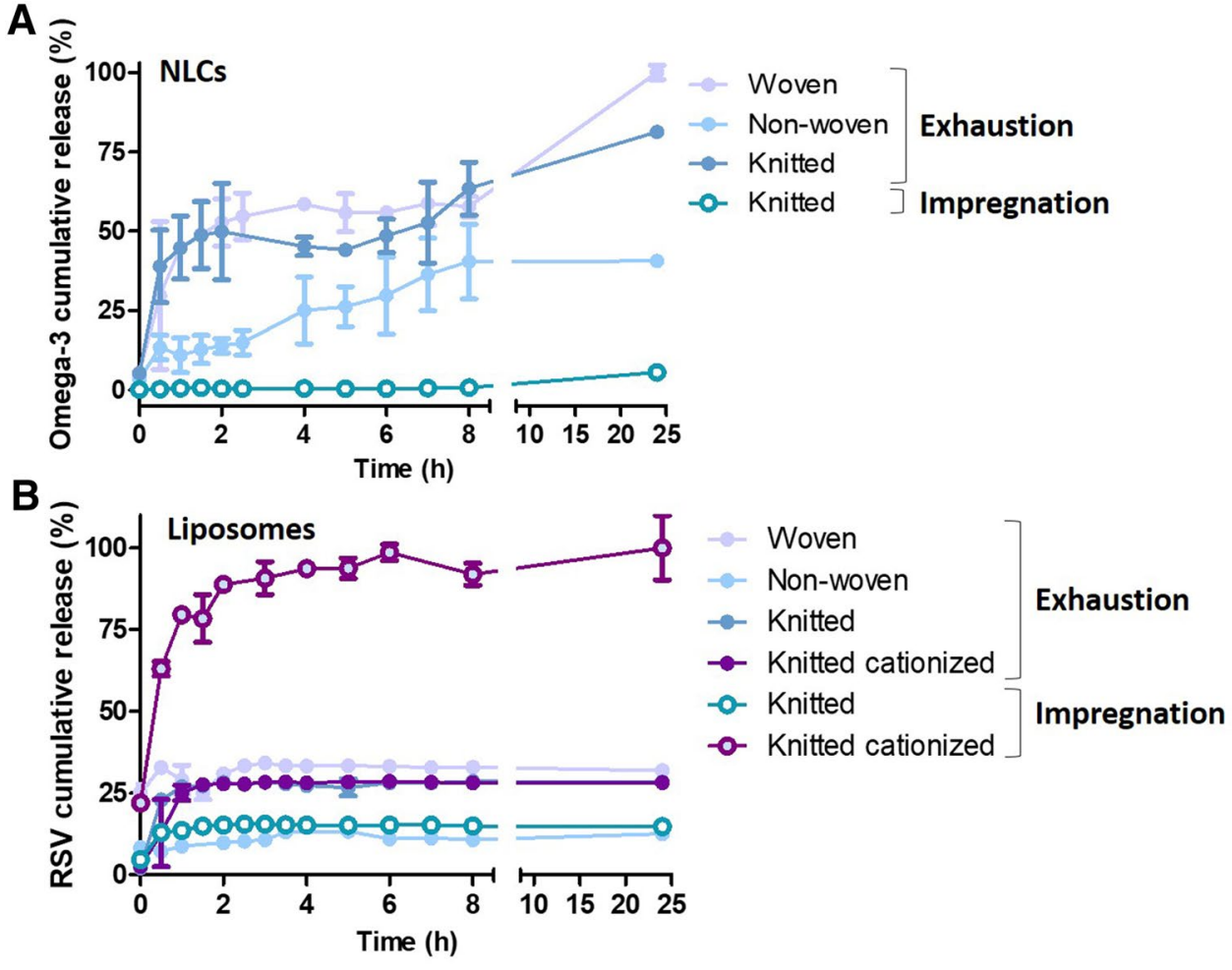

last $16 \mathrm{~h}$ of the test, omega- 3 release still occurred so it is important to extend this experiment to understand when the maximum release is reached.

With the non-woven fabric, omega-3 suffered a small burst release during the first $8 \mathrm{~h}$. After $24 \mathrm{~h}$, the amount of omega- 3 released is similar to the observed after the first $8 \mathrm{~h}$ (Fig. 5a). Therefore, it can be concluded that $8 \mathrm{~h}$ is enough time to achieve the maximum release from nonwoven fabric.

Despite being the one with higher mass gain (Fig. 1a), non-woven fabric had a lower amount of omega-3 released (Fig. 5a), comparing with woven and knitted fabrics functionalized by exhaustion. This can be explained by the macrostructure of this fabric which makes the contact 
with the release medium more difficult when compared with the flat structures (woven and knitted fabrics).

The impregnated knitted fabric presented very low amounts of omega- 3 release. This proves the weak functionalization using this technique, confirming the results obtained by mass gain (Fig. 1a) and FTIR-ATR spectra (Fig. 2b).

In summary, all textiles functionalized with NLCs by exhaustion presented a fast omega- 3 release suggesting that these textiles are suitable as wound dressing for acute wounds. To conclude about the suitability of these textile in chronic wounds, a more extended release study is mandatory. Woven fabric functionalized with NLCs by exhaustion appears to be the most promising substrate since it presented the higher amount of released omega-3 during the $24 \mathrm{~h}$ test.

Almost all substrates functionalized with liposomes had a similar release profile with a maximum of RSV being released in the first hour (Fig. 5b). Cationized knitted fabric functionalized by impregnation of liposomes was the only exception. The higher affinity of negative charged liposomes to the cationized knitted fabric can explain the prolongated release profile. Moreover, in this substrate, not only the amount of RSV released was higher, but the maximum released was only reached after $6 \mathrm{~h}$. The higher amount of RSV released is in accordance with the mass gain results. In comparison with other substrates and except from the non-woven fabric, the cationized knitted fabric showed the highest mass gain obtained (Fig. 1b).

In conclusion, knitted fabric cationized and functionalized by impregnation is the most promising technique to functionalize textiles with liposomes, since the amount of RSV released from this textile is higher and more prolonged.

\section{Conclusions}

The amount of NLCs and liposomes adsorbed was related with textile substrates adsorption capacity, being the nonwoven textile the most efficient. The exhaustion technique was more suitable for NLCs incorporation whereas the impregnation technique was adequate for liposomes. The FTIR-ATR and DSC profiles confirmed the NLCs presence on the textile samples. Otherwise, no significant differences were observed between FTIR-ATR and DSC profiles samples functionalized with liposomes. Lipid nanosystems maintained the textiles permeability to water vapour. Moreover, functionalization of the textiles did not affect the permeability to air. Both characteristics are favorable for the healing process. Lipid nanosystems also present an inhibitory effect of NO production, regarded as important to combat inflammation and promote tissue healing.
The woven fabric functionalized by exhaustion with NLCs was the most promising for wound dressings application, since it had the most suitable omega- 3 release profile (i.e., the higher amount released). Regarding the liposome's application, the most promising textile was the cationized knitted fabric functionalized by impregnation, since it was the substrate capable of retaining more RSV and showed a more prolongated release profile of this bioactive.

In conclusion, lipid nanosystems, such as NLCs and liposomes, can be used to improve textiles and enhance their application for anti-inflammatory effects in wound healing.

Acknowledgements This work is financed by Project UID/ CTM/00264/2019 of 2C2T - Centro de Ciência e Tecnologia Têxtil, funded by National Founds through FCT/MCTES. The authors also acknowledge the Portuguese Foundation for Science and Technology (FCT) for financial support in the framework of the Strategic Funding UID/Multi/04546/2013 and UID/FIS/04650/2019 in the ambit of the project POCl-01-0145-FEDER-032651, co-financed by the European Regional Development Fund (ERDF), through COMPETE 2020, under Portugal 2020.

\section{Compliance with ethical standards}

Conflict of interest On behalf of all authors, the corresponding author states that there is no conflict of interest.

\section{References}

1. Pinho E, Soares $G$ (2018) Functionalization of cotton cellulose for improved wound healing. J Mater Chem B 6:1887-1898. https ://doi.org/10.1039/C8TB00052B

2. McDaniel JC, Belury M, Ahijevych K, Blakely W (2008) $\omega-3$ fatty acids effect on wound healing. Wound Repair Regen 16(3):337345. https://doi.org/10.1111/j.1524-475X.2008.00388.x

3. Shrestha H, Bala R, Arora S (2014) Lipid-based drug delivery systems. J Pharm. https://doi.org/10.1155/2014/801820

4. Serfert Y, Drusch S, Schwarz K (2010) Sensory odour profiling and lipid oxidation status of fish oil and microencapsulated fish oil. Food Chem 123(4):968-975. https://doi.org/10.1016/j.foodc hem.2010.05.047

5. Yaman I, Derici H, Kara C, Kamer E, Diniz G, Ortac R, Sayin O (2013) Effects of resveratrol on incisional wound healing in rats. Surg Today 43(12):1433-1438. https://doi.org/10.1007/s0059 5-012-0455-7

6. Zhao P, Sui B, Liu N, Lv Y, Zheng C, Lu Y, Huang W, Zhou C, Chen J, Pang D, Fei D, Xuan K, Hu C, Jin Y (2017) Anti-aging pharmacology in cutaneous wound healing: effects of metformin, resveratrol, and rapamycin by local application. Aging Cell 16(5):10831093. https://doi.org/10.1111/acel.12635

7. Wenzel E, Somoza V (2005) Metabolism and bioavailability of trans-resveratrol. Mol Nutr Food Res 49(5):472-481. https://doi. org/10.1002/mnfr.200500010

8. Neves AR, Lúcio M, Lima JLC, Reis S (2012) Resveratrol in medicinal chemistry: a critical review of its pharmacokinetics, drug-delivery, and membrane interactions. Curr Med Chem 19(11):1663-1681. https://doi.org/10.2174/092986712799945 085 
9. Barbosa C, Santos-Pereira C, Soares I, Martins V, Terra-Matos J, Côrte-Real M, Lúcio M, Oliveira MECDR, Gerós H (2019) Resveratrol-loaded lipid nanocarriers are internalized by endocytosis in yeast. J Nat Prod 5:1240-1249. https://doi.org/10.1021/acs. jnatprod.8b01003

10. Tang L, Gao J, Chen X, Xie X (2006) Inhibitory effect of resveratrol on the proliferation of synoviocytes in rheumatoid arthritis and its mechanism in vitro. J Cent South Univ Med Sci 31(4):528-533

11. Švajger U, Jeras M (2012) Anti-inflammatory effects of resveratrol and its potential use in therapy of immune-mediated diseases. Int Rev Immunol 31(3):202-222. https://doi.org/10.3109/08830 185.2012.665108

12. MacMicking J, Xie Q, Nathan C (1997) Nitric oxide and macrophage function. Annu Rev Immunol 15:323-350. https://doi. org/10.1146/annurev.immunol.15.1.323

13. Hierholzer C, Harbrecht B, Menezes JM, Kane J, MacMicking J, Nathan CF, Peitzman AB, Billiar TR, Tweardy DJ (1998) Essential role of induced nitric oxide in the initiation of the inflammatory response after hemorrhagic shock. J Exp Med 187(6):917-928. https://doi.org/10.1084/jem.187.6.917

14. Sobral F, Sampaio A, Falcão S, Queiroz MJ, Calhelha RC, VilasBoas M, Ferreira IC (2016) Chemical characterization, antioxidant, anti-inflammatory and cytotoxic properties of bee venom collected in Northeast Portugal. Food Chem Toxicol 94:172-177. https://doi.org/10.1016/j.fct.2016.06.008

15. Atiyeh BS, loannovich J, Al-Amm CA, El-Musa KA (2002) Management of acute and chronic open wounds: the importance of moist environment in optimal wound healing. Curr Pharm Biotechnol 3(3):179-195. https://doi.org/10.2174/1389201023 378283

16. Hinman CD, Maibach H (1963) Effect of air exposure and occlusion on experimental human skin wounds. Nature 200:377-378. https://doi.org/10.1038/200377a0

17. Lopes CM, Silva J, Oliveira MECDR, Lúcio M (2018) Lipid-based colloidal carriers for topical application of antiviral drugs. In: Grumezescu AM (ed) Design of nanostructures for versatile therapeutic applications. Elsevier, Amsterdam, pp 565-622

18. Ruelle $P(2000)$ The n-octanol and n-hexane/water partition coefficient of environmentally relevant chemicals predicted from the mobile order and disorder (MOD) thermodynamics. Chemosphere 40(5):457-512. https://doi.org/10.1016/S0045 -6535(99)00268-4

19. Augustin MA, Sanguansri L, Head R (2003) Gl tract delivery systems. Patent WPO 2003906417. https://patents.google.com/ patent/WO2005048998A1. Accessed 13 Mar 2019

20. De Leeuw J, De Vijlder H, Bjerring P, Neumann HA (2009) Liposomes in dermatology today. J Eur Acad Dermatol Venereol 23(5):505-516. https://doi.org/10.1111/j.1468-3083.2009.03100 .x

21. Hadgraft J (2004) Skin deep. Eur J Pharm Biopharm 58(2):291299. https://doi.org/10.1016/j.ejpb.2004.03.002

22. Hamblin MR, Avci P, Prow TW (2016) Nanoscience in dermatology. Academic Press, Boston

23. Badran M (2014) Formulation and in vitro evaluation of flufenamic acid loaded deformable liposomes for improved skin delivery. Dig J Nanomater Biostruct 9(1):83-91

24. Chen M, Liu X, Fahr A (2011) Skin penetration and deposition of carboxyfluorescein and temoporfin from different lipid vesicular systems: in vitro study with finite and infinite dosage application. Int J Pharm 408(1-2):223-234. https://doi.org/10.1016/j. ijpharm.2011.02.006

25. Putri DCA, Dwiastuti R, Marchaban M, Nugroho AK (2017) Optimization of mixing temperature and sonication duration in liposome preparation. J Pharm Sci Community 14(2):79-85. https:// doi.org/10.24071/jpsc. 142728
26. Benson HAE, Mohammed Y, Grice JE, Roberts MS (2016) Formulation effects on topical nanoparticle penetration. In: Hamblin MR, Avci P, Prow TW (eds) Nanoscience in dermatology. Academic Press, Amsterdam, pp 115-126

27. Tucker IM, Corbett JCW, Fatkin J, Jack RO, Kaszuba M, MacCreath B, McNeil-Watson F (2015) Laser doppler electrophoresis applied to colloids and surfaces. Curr Opin Colloid Interface Sci 20(4):215-226. https://doi.org/10.1016/j.cocis.2015.07.001

28. Uskoković V (2012) Dynamic light scattering based microelectrophoresis: main prospects and limitations. J Dispers Sci Technol 33(12):1762-1786. https://doi.org/10.1080/01932 691.2011 .625523

29. Kheradmandnia S, Vasheghani-Farahani E, Nosrati M, Atyabi F (2010) Preparation and characterization of ketoprofen-loaded solid lipid nanoparticles made from beeswax and carnauba wax. Nanomedicine 6(6):753-759. https://doi.org/10.1016/j. nano.2010.06.003

30. Koh TJ, DiPietro LA (2011) Inflammation and wound healing: the role of the macrophage. Expert Rev Mol Med 13:e23. https ://doi.org/10.1017/S1462399411001943

31. Khanna S, Biswas S, Shang Y, Collard E, Azad A, Kauh C, Bhasker V, Gordillo GM, Sen CK, Roy S (2010) Macrophage dysfunction impairs resolution of inflammation in the wounds of diabetic mice. PLoS ONE 5(3):e9539. https://doi.org/10.1371/journ al.pone.0009539\%0A

32. Acosta JB, del Barco DG, Vera DC, Savigne W, Lopez-Saura P, Nieto GG, Schultz GS (2008) The pro-inflammatory environment in recalcitrant diabetic foot wounds. Int Wound J 5(4):530-539. https://doi.org/10.1111/j.1742-481X.2008.00457

33. Sharma JN, Al-Omran A, Parvathy SS (2007) Role of nitric oxide in inflammatory diseases. Inflammopharmacology 15(6):252259. https://doi.org/10.1007/s10787-007-0013-x

34. Lima TM, Sa Lima L, Scavone C, Curi R (2006) Fatty acid control of nitric oxide production by macrophages. FEBS Lett 580(13):3287-3295. https://doi.org/10.1016/j.febsl et.2006.04.091

35. Kimbrough CW, Lakshmanan J, Matheson PJ, Woeste M, Gentile A, Benns MV, Zhang B, Smith JW, Harbrecht BG (2015) Resveratrol decreases nitric oxide production by hepatocytes during inflammation. Surgery 158(4):1095-1101. https://doi. org/10.1016/j.surg.2015.07.012

36. Xiong Q, Tezuka Y, Kaneko T, Li H, Tran LQ, Hase K, Namba T, Kadota S (2000) Inhibition of nitric oxide by phenylethanoids in activated macrophages. Eur J Pharmacol 400(1):137-144. https://doi.org/10.1016/S0014-2999(00)00354-X

37. Jass J, Tjärnhage T, Puu G (2000) From liposomes to supported, planar bilayer structures on hydrophilic and hydrophobic surfaces: an atomic force microscopy study. Biophys J 79(6):31533163. https://doi.org/10.1016/S0006-3495(00)76549-0

38. Nunes C, Brezesinski G, Lopes D, Lima JLFC, Reis S, Lúcio M (2011) Lipid-drug interaction: biophysical effects of tolmetin on membrane mimetic systems of different dimensionality. J Phys Chem B 115(43):12615-12623. https://doi.org/10.1021/ jp206013z

39. Makled S, Nafee N, Boraie N (2017) Nebulized solid lipid nanoparticles for the potential treatment of pulmonary hypertension via targeted delivery of phosphodiesterase-5-inhibitor. Int J Pharm 517(1-2):312-321. https://doi.org/10.1016/j.ijpha rm.2016.12.026

40. Sade A, Banerjee S, Severcan F (2010) Concentration-dependent differing actions of the nonsteroidal anti-inflammatory drug, celecoxib, in distearoyl phosphatidylcholine multilamellar vesicles. J Liposome Res 20(2):168-177. https://doi. org/10.3109/08982100903244492 
41. Blume A (1996) Properties of lipid vesicles: FT-IR spectroscopy and fluorescence probe studies. Curr Opin Colloid Interface Sci 1(1):64-77. https://doi.org/10.1016/S1359-0294(96)80046-X

42. Zhang J (2009) Analysis and characterization of consumer products by FTIR, Raman, Chemometrics, and two dimensional ATRFTIR correlation spectroscopy. Dissertation, Graduate SchoolNew Brunswick Rutgers, the State University of New Jersey

43. Chung C, Lee M, Choe EK (2004) Characterization of cotton fabric scouring by FT-IR ATR spectroscopy. Carbohydr Polym 58(4):417-420. https://doi.org/10.1016/j.carbpol.2004.08.005

44. Pujara N, Jambhrunkar S, Wong KY, McGuckin M, Popat A (2017) Enhanced colloidal stability, solubility and rapid dissolution of resveratrol by nanocomplexation with soy protein isolate. J Colloid Interface Sci 488:303-308. https://doi.org/10.1016/j. jcis.2016.11.015

45. Pinho E, Henriques M, Oliveira R, Dias A, Soares G (2010) Development of biofunctional textiles by the application of resveratrol to cotton, bamboo, and silk. Fibers Polym 11(2):271-276. https://doi.org/10.1007/s12221-010-0271-x

46. Hartzell-Lawson MM, Hsieh Y (2000) Characterizing the noncellulosics in developing cotton fibers. Text Res J 70(9):810-819. https://doi.org/10.1177/004051750007000909

47. Scacchetti F, Pinto E, Soares G (2017) Functionalization and characterization of cotton with phase change materials and thyme oil encapsulated in beta-cyclodextrins. Prog Org Coat 107:64-74. https://doi.org/10.1016/j.porgcoat.2017.03.015

48. Avasatthi V, Pawar H, Dora CP, Bansod P, Gill MS, Suresh S (2016) A novel nanogel formulation of methotrexate for topical treatment of psoriasis: optimization, in vitro and in vivo evaluation. Pharm Dev Technol 21(5):554-562. https://doi. org/10.3109/10837450.2015.1026605

49. Wang JJ, Liu KS, Sung KC, Tsai CY, Fang JY (2009) Skin permeation of buprenorphine and its ester prodrugs from lipid nanoparticles: lipid emulsion, nanostructured lipid carriers and solid lipid nanoparticles. J Microencapsul 26(8):734-747. https://doi. org/10.3109/02652040902746679

50. Sebedio JL, Ratnayake WMN, Ackman RG, Prevost J (1993) Stability of polyunsaturated omega-3 fatty acids during deep fat frying of Atlantic mackerel (Scomber scombrus L.). Food Res Int 26(3):163-172. https://doi.org/10.1016/0963-9969(93)90049-O

51. Ajmeri JR, Ajmeri CJ (2011) Nonwoven materials and technologies for medical applications. In: Bartels VT (ed) Handbook of medical textiles. Elsevier, Amsterdam, pp 106-131

52. Xu R, Xia H, He W, Li Z, Zhao J, Liu B, Wang Y, Lei Q, Kong Y, Bai Y, Yao Z, Yan R, Li H, Zhan R, Yang S, Luo G, Wu J (2016) Controlled water vapor transmission rate promotes wound-healing via wound re-epithelialization and contraction enhancement. Sci Rep 6(1):24596. https://doi.org/10.1038/srep24596

Publisher's Note Springer Nature remains neutral with regard to jurisdictional claims in published maps and institutional affiliations. 